\title{
Existence of solutions for nonlocal boundary value problem for Caputo nonlinear fractional differential inclusion
}

\author{
Noureddine Bouteraa ${ }^{a^{*}}$ and Slimane Benaicha ${ }^{b}$ \\ ${ }^{a}$ Laboratory of Fundamental and Applied Mathematics of Oran (LMFAO), University of Oran1, Ahmed Benbella. Algeria \\ ${ }^{\mathrm{b}}$ Laboratory of Fundamental and Applied Mathematics of Oran (LMFAO), University of Oranl, Ahmed Benbella. Algeria \\ *Corresponding author E-mail: bouteraa-27@hotmail.fr
}

\author{
Article Info \\ Keywords: Existence, Fractional differ- \\ ential inclusion, Fixed point theorem, \\ Nonlocal boundary \\ 2010 AMS: 34A60, 34B15 \\ Received: 12 April 2018 \\ Accepted: 8 May 2018 \\ Available online: 27 May 2018
}

\begin{abstract}
This paper deals with the existence of solutions for nonlinear fractional differential inclusions supplemented with three-point boundary conditions. First, we investigate it for $L^{1}$ Caratheodory convex-compact valued multifunction. Then, we investigate it for nonconvexcompact valued multifunction via some conditions. Two illustrative examples are presented at the end of the paper to illustrate the validity of our results.
\end{abstract}

\section{Introduction}

The concept of fractional calculus has played an important role in improving the work based on integer-order (classical) calculus in several diverse disciplines of science and engineering. In fact, quantum calculus has a rich history and the details of this basic notions, results and methods can be found in the text $[2,26,37]$ and papers $[10,22]$. The nonlocal nature of a fractional order differential operator, which take into account hereditary properties of various material and processes, has helped to improve the mathematical modeling of many natural phenomena and physical processes, see for example [4, 5, 21]. The increasing interest of fractional differential equations and inclusions are motivated by their applications in various fields of science such as physics chemistry, biology, economics, fluid mechanics, control theory, etc, we refer the reader to $[9,17,30]$ and the references therein. Realistic problems arising from economics, optimal control, stochastic analysis can be modelled as differential inclusion. So much attention has been paid by many authors to study this kind of problems, see [4, 5, 36]. On the other hand boundary value problems with local and nonlocal boundary conditions constitute a very interesting and important class of problems. They include two, three and multipoint boundary value problems. The existence and multiplicity of positive solutions for such problems have received a great deal of attentinos. To identify a few, we refer the reader to [8, 11, 13, 18, 19, 20, 24, 25, 27, 28, 29, 31, 32, 33, 34].

In this paper, we are interested in the existence of solutions for the Caputo fractional differential inclusion

$$
{ }^{c} D^{\alpha} u(t) \in F\left(t, u(t), u^{\prime}(t)\right), \quad t \in J=[0,1],
$$

subject to three-point boundary conditions

$$
\left\{\begin{array}{l}
\beta u(0)+\gamma u(1)=u(\eta), \\
u(0)=\int_{0}^{\eta} u(s) d s \\
\beta^{c} D^{p} u(0)+\gamma^{c} D^{p} u(1)={ }^{c} D^{p} u(\eta),
\end{array}\right.
$$

where $2<\alpha \leq 3,1<p \leq 2,0<\eta<1, \beta, \gamma \in \mathbb{R}^{+}, f \in C\left([0,1] \times \mathbb{R}^{2}, \mathbb{R}\right)$ and ${ }^{c} D^{\alpha}$ denotes the Caputo fractional derivative of order $\alpha$.

The current paper is organized as follows. In section 2, we introduce some definitions and preliminary results that will be used in the remainder of the paper. In section 3, we present existence results for the problem (1.1) - (1.2) when the right-hand side is convex-compact as well as nonconvex-compact values. In the first result we use the fixed-point theorem (Lemma 2.12) for multivalued maps (see [3]) while in the second result we prove the existence by applying a fixed-point theorem for contraction multivalued maps due to Covitz and Nadler and we give two examples to illustrate our results. 


\section{Preliminaries}

In this section, we introduce some necessary definitions and lemmas of fractional calculus to facilitate the analysis of the problem (1.1) - (1.2). These details can be found in the recent literature; see $[1,12,16]$ and the references therein.

Definition 2.1. Let $\alpha>0, n-1<\alpha<n, n=[\alpha]+1$ and $u \in C([0, \infty), \mathbb{R})$. The Caputo derivative of fractional order $\alpha$ for the function $u$ is defined by

$$
{ }^{c} D^{\alpha} u(t)=\frac{1}{\Gamma(n-\alpha)} \int_{0}^{t}(t-s)^{n-\alpha-1} u^{(n)}(s) d s .
$$

where $\Gamma(\cdot)$ is the Euler gamma function.

Definition 2.2. The Riemann-Liouville fractional integral of order $\alpha>0$ of a function $u:(0, \infty) \rightarrow \mathbb{R}$ is given by

$$
I^{\alpha} u(t)=\frac{1}{\Gamma(\alpha)} \int_{0}^{t}(t-s)^{\alpha-1} u(s) d s, \quad t>0,
$$

where $\Gamma(\cdot)$ is the Euler gamma function, provided that the right side is pointwise defined on $(0, \infty)$.

Lemma 2.3. [22] Let $\alpha, \beta \geq 0$ and $u \in L^{p}(0,1), 0 \leq p \leq+\infty$. Then the next formulas hold.

(i) $\left(I^{\beta} I^{\alpha} u\right)(t)=I^{\alpha+\beta} u(t)$,

(ii) $\left(D^{\beta} I^{\alpha} u\right)(t)=I^{\alpha-\beta} u(t)$,

(iii) $\left(D^{\alpha} I^{\alpha} u\right)(t)=u(t)$.

Lemma 2.4. [26] Let $\alpha>0, n-1<\alpha<n$ and the function $g:[0, T] \rightarrow \mathbb{R}$ be continuous for each $T>0$. Then, the general solution of the fractional differential equation ${ }^{c} D^{\alpha} g(t)=0$ is given by

$$
g(t)=c_{0}+c_{1} t+\cdots+c_{n-1} t^{n-1},
$$

where $c_{0}, c_{1}, \ldots, c_{n-1}$ are real constants and $n=[\alpha]+1$.

Lemma 2.5. [4] Assume that $u \in C[0,1] \cap L^{1}(0,1)$ with a Caputo fractional derivative of order $\alpha>0$ that belongs to $u \in C^{n}[0,1]$, then

$$
I^{\alpha c} D^{\alpha} u(t)=u(t)+c_{0}+c_{1} t+\cdots+c_{n-1} t^{n-1},
$$

where $c_{0}, c_{1}, \ldots, c_{n-1}$ are real constants and $n=[\alpha]+1$.

We will present notations, definitions and preliminary facts from multivalued analysis which are used throughout this paper. Here $(C[0,1], \mathbb{R})$ denotes the Banach space of all continuous functions from $[0,1]$ into $\mathbb{R}$ with the norm $\|u\|=\sup \{|u(t)|:$ for all $t \in[0,1]\}, L^{1}([0,1], \mathbb{R})$, the Banach space of measurable functions $u:[0,1] \rightarrow \mathbb{R}$ which are Lebesgue integrable, normed by $\|u\|_{L^{1}}=\int_{0}^{1}|u(t)| d t$, and $A C^{i}([0,1], \mathbb{R})$ the space of $i$-times differentiable functions $u:[0,1] \rightarrow \mathbb{R}$ whose $i-t h$ derivative $u^{(i)}$ is absolutely continuous.

Let $(X, d)$ be a metric space induced from the normed space $(X,\|\cdot\|)$. We denote

$$
\begin{aligned}
& P_{0}(X)=\{A \in P(X): A \neq \phi\}, \\
& P_{b}(X)=\left\{A \in P_{0}(X): \text { A is bounded }\right\}, \\
& P_{c l}(X)=\left\{A \in P_{0}(X): \text { A is closed }\right\}, \\
& P_{c p, c v}(X)=\left\{A \in P_{0}(X): \text { A is compact and convex }\right\}, \\
& P_{b, c l}(X)=\left\{A \in P_{0}(X): \text { A isclosed and bounded }\right\} .
\end{aligned}
$$

Definition 2.6. A multivalued maps $G: X \rightarrow P(X)$.

(1) is convex (closed) valued if $G(X)$ is convex (closed) for all $u \in X$,

(2) is bounded on bounded sets if $G(B)=\cup_{u \in B} G(u)$ is bounded in $X$ for all $B \in P_{b}(X)$ i.e. $\sup _{u \in B}\{\sup \{|v|, v \in G(u)\}\}<\infty$,

(3) is called upper semicontinuous (u.s.c) on $X$ if for each $u_{0} \in X$, the set $G\left(u_{0}\right)$ is a nonempty closed subset of $X$ and iffor each open set $N$ of $X$ containing $G\left(u_{0}\right)$ there exists an open neighborhood $N_{0}$ of $u_{0}$ such that $G\left(N_{0}\right) \subseteq N$,

(4) is said to be completely continuous if $G(B)$ is relatively compact for every $B \in P_{b}(X)$,

(5)has a fixed point if there is $u \in X$ such that $u \in G(X)$. The fixed point set of the multi-valued operator $G$ will be denote by Fix $G$.

Remark 2.7. It is well known that, if the multi-valued map $G$ is completely continuous with nonempty compact values, then $G$ is u.s.c if and only if $G$ has closed graph i.e., $u_{n} \rightarrow u, v_{n} \rightarrow v, v_{n} \in G\left(u_{n}\right)$ imply $v \in G(u)$. 
Definition 2.8. A multi-valued maps $G:[0,1] \rightarrow P_{c l}(\mathbb{R})$ is said to be measurable if for every $y \in \mathbb{R}$ the function

$$
t \longmapsto d(y, G(t))=\inf \{\|y-z\|: z \in G(t)\},
$$

is measurable.

Definition 2.9. A multi-valued maps $F:[0,1] \times \mathbb{R} \times \mathbb{R} \rightarrow 2^{\mathbb{R}}$ is said to be Caratheodory if,

(i) $t \longmapsto F(t, u, v)$ for all $u, v \in \mathbb{R}$,

(ii) $t \longmapsto F(t, u, v)$ is upper semi-continuous for almost all $t \in[0,1]$. Further a Caratheodory function is called $L^{1}-$ Caratheodory,

(iii) for each $\rho>0$, there exists $\phi_{\rho} \in L^{1}\left([0,1], \mathbb{R}^{+}\right)$such that $\|F(t, u, v)\|=\sup \{|v|, v \in F(t, u, v)\} \leq \phi_{\rho}(t)$, for all $|u|,|v|<\rho$.

Definition 2.10. Let $Y$ be a nonempty closed subset of a Banach spase $E$ and $G: Y \rightarrow P_{c l}(E)$ be a multivalued operator with nonempty closed values. $G$ is said to be lower semicontinuous (l.s.c) if the set $\{x \in X: G(x) \cap U \neq \phi\}$ is open for any open set $U$ in $E$.

For $y \in(C[0,1], \mathbb{R})$, define the set of selection of $F$ by

$$
S_{F, u}=\left\{v \in A C([0,1], \mathbb{R}), v \in F\left(t, u(t), u^{\prime}(t)\right), \text { for almost all } t \in[0,1]\right\} .
$$

For $P(X)=2^{X}$, consider the Pompeiu-Hausdorff metric (see[?])

$H_{d}: 2^{\mathbb{X}} \times 2^{\mathbb{X}} \rightarrow[0, \infty)$ given by

$$
H_{d}(A, B)=\max \left\{\operatorname{supd}_{a \in A}(a, B), \operatorname{supd}_{b \in B}(b, A)\right\},
$$

where $d(a, B)=\inf _{b \in B} d(a, b)$ and $d(b, A)=\underset{a \in A}{\inf d}(a, b)$. Then $\left(P_{b, c l}(X), H_{d}\right)$ is a metric space and $\left(P_{c l}(X), H_{d}\right)$ is a generalized metric space see [7].

Let $Y$ be a nonempty closed subset of a Banach spase $E$ and $G: Y \rightarrow P_{c l}(E)$ be a multivalued operator with nonempty closed values. $G$ is said to be lower semicontinuous (1.s.c) if the set $\{x \in X: G(x) \cap U \neq \phi\}$ is open for any open set $U$ in $E$.

$G$ has a fixed point if there is $x \in Y$ such that $x \in G(x)$. For more details on the multi-valued maps, see the book of Aubin and Celina [14], Demling [15], Gorniewicz [16] and Hu and Papageorgiou [35].

Lemma 2.11. [1] Let $X$ be a Banach space. $F:[0,1] \times \mathbb{X} \rightarrow P_{c p, c v}(X)$ an $L^{1}-$ Caratheodory multifunction and $\Theta$ a linear continuous mapping from $L^{1}([0,1], X)$ to $C([0,1], X)$. Then the operator $\left(\Theta \circ S_{F}\right)(u)=\Theta\left(S_{F, u}\right)$ is a closed graph operator in $C([0,1], X) \times$ $C([0,1], X)$.

Lemma 2.12. [3] Let $E$ be a Banach space. $C$ a closed convex subset of $E, U$ an open subset of $C$ and $0 \in U$. Suppose that $F: \bar{U} \rightarrow P_{c p, c v}(C)$ is an upper semi-continuous compact map, where $P_{c p, c v}(C)$ denotes the family of nonempty, compact convex subset of $C$. Then either $F$ has a fixed point in $\bar{U}$ or there exist $u \in \partial U$ and $\lambda \in(0,1)$ such that $u \in \lambda F(U)$.

Lemma 2.13. [12] A multifunction $F: X \rightarrow C(X)$ is called a contraction whenever there exists $\gamma \in(0,1)$ such that $H_{d}(N(u), N(v)) \leq$ $\gamma d(u, v)$ for all $u, v \in X$

Lemma 2.14. ( Covitz-Nadler) Let $(X, d)$ be a complete metric space. If $N: X \rightarrow P_{c l}(X)$ is a contraction, then FixN $\neq \emptyset$.

\section{Existence results}

Let $X=\left\{u: u, u^{\prime} \in C([0,1], \mathbb{R})\right\}$ endowed with the norm defined by $\|u\|=\sup _{t \in[0,1]}|u(t)|+\sup _{t \in[0,1]}\left|u^{\prime}(t)\right|$ such that $\|u\|<\infty$. Then $(X,\|\cdot\|)$ is a Banach space.

Lemma 3.1. Let $y \in C([0,1], \mathbb{R})$. Then the integral solution of the linear fractional differential equation

$$
{ }^{c} D^{\alpha} u(t)=y(t) \quad t \in[0,1], \alpha \in(2,3],
$$

subject to three-point boundary conditions

$$
\begin{gathered}
\beta u(0)+\gamma u(1)=u(\eta), \quad \beta \geq 0, \gamma \geq 0, \\
u(0)=\int_{0}^{\eta} u(s) d s, \quad \eta \in(0,1), \\
\beta^{c} D^{p} u(0)+\gamma^{c} D^{p} u(1)={ }^{c} D^{p} u(\eta), \quad p \in(1,2],
\end{gathered}
$$

is given by

$$
\begin{aligned}
u(t)= & \int_{0}^{t} \frac{(t-s)^{\alpha-1}}{\Gamma(\alpha)} y(s) d s+\frac{1}{1-\eta} \int_{0}^{\eta}\left(\int_{0}^{s} \frac{(s-\tau)^{\alpha-1}}{\Gamma(\alpha)} y(\tau) d \tau\right) d s-\frac{\Lambda_{1}(t)}{Q_{1}(1-\eta)} \int_{0}^{\eta}\left(\int_{0}^{s} \frac{(s-\tau)^{\alpha-1}}{\Gamma(\alpha)} y(\tau) d \tau\right) d s \\
& -\frac{\Lambda_{2}(t) M_{1}}{6(1-\eta) Q_{1}}\left[\int_{0}^{\eta} \frac{(\eta-s)^{\alpha-p-1}}{\Gamma(\alpha-p)} y(s) d s-\gamma \int_{0}^{1} \frac{(1-s)^{\alpha-p-1}}{\Gamma(\alpha-p)} y(s) d s\right] \\
& +\frac{\Lambda_{1}(t)}{Q_{1}(\beta+\gamma-1)}\left[\int_{0}^{\eta} \frac{(\eta-s)^{\alpha-1}}{\Gamma(\alpha)} y(s) d s-\gamma \int_{0}^{1} \frac{(1-s)^{\alpha-1}}{\Gamma(\alpha)} y(s) d s\right]
\end{aligned}
$$


where

$$
\begin{gathered}
\Lambda_{1}(t)=(\beta+\gamma-1)\left(\eta^{2}+2(1-\eta) t\right), M_{1}=\frac{\Gamma(3-p)}{\gamma-\eta^{2-p}} \\
\Lambda_{2}(t)=\left(\eta^{3}(\beta+\gamma-1)+3\left(\gamma-\eta^{2}\right)(1-\eta)\right)\left(\eta^{2}+2(1-\eta) t\right)-Q_{1}\left(\eta^{3}+3(1-\eta) t^{2}\right),
\end{gathered}
$$

and

$$
Q_{1}=2(1-\eta)(\gamma-\eta)+\eta^{2}(\beta+\gamma-1) \neq 0 .
$$

Proof. In view of Lemma 2.3 and Lemma 2.5, the solution of equation (3.1) can be written as

$$
u(t)=I^{\alpha} y(t)+c_{0}+c_{1} t+c_{2} t^{2}=\int_{0}^{t} \frac{(t-s)^{\alpha-1}}{\Gamma(\alpha)} y(s) d s+c_{0}+c_{1} t+c_{2} t^{2},
$$

where $c_{0}, c_{1}, c_{2} \in \mathbb{R}$ are arbitrary constants.

Differentiating both sides of (3.6) and applying Definition 2.1, Lemma 2.3 and Lemma 2.5, we obtain

$$
{ }^{c} D^{p} u(t)=I^{\alpha-p} y(t)+c_{2} \frac{2 t^{2-p}}{\Gamma(3-p)}=\int_{0}^{t} \frac{(t-s)^{\alpha-p-1}}{\Gamma(\alpha-p)} y(s) d s+\frac{2 t^{2-p}}{\Gamma(3-p)} c_{2},
$$

where $\alpha \in(2,3]$ and $p \in(1,2]$.

Integrating both sides of (3.6), we obtain

$$
\int_{0}^{\eta} u(t) d t=\int_{0}^{\eta}\left(\int_{0}^{t} \frac{(t-s)^{\alpha-1}}{\Gamma(\alpha)} y(s) d s\right) d t+c_{0} \eta+\frac{1}{2} c_{1} \eta^{2}+\frac{1}{3} c_{2} \eta^{3}
$$

By using the boundary condition (3.2) in (3.6), we obtain

$$
c_{0}(\beta+\gamma-1)+c_{1}(\gamma-\eta)+c_{2}\left(\gamma-\eta^{2}\right)=\int_{0}^{\eta} \frac{(\eta-s)^{\alpha-1}}{\Gamma(\alpha)} y(s) d s-\gamma \int_{0}^{1} \frac{(1-s)^{\alpha-1}}{\Gamma(\alpha)} y(s) d s
$$

By using the boundary condition (3.3) in (3.6) and (3.8), we obtain

$$
(1-\eta) c_{0}-\int_{0}^{\eta} \frac{(\eta-s)^{\alpha-1}}{\Gamma(\alpha)} y(s) d s-\frac{1}{2} c_{1} \eta^{2}-\frac{1}{3} \eta^{3}=0 .
$$

By using the boundary condition (3.4) in (3.7), we obtain

$$
c_{2}=\frac{M_{1}}{2}\left(\int_{0}^{\eta} \frac{(\eta-s)^{\alpha-p-1}}{\Gamma(\alpha-p)} y(s) d s-\gamma \int_{0}^{1} \frac{(1-s)^{\alpha-p-1}}{\Gamma(\alpha-p)} y(s) d s\right) .
$$

Solving the above system of the equations (3.9), (3.10) and (3.11) for $c_{0}, c_{1}, c_{2}$, we get

$$
\begin{aligned}
c_{2}=\frac{M_{1}}{2}\left(I^{\alpha-p} y(\eta)-\gamma I^{\alpha-p} y(1)\right)=\frac{M_{1}}{2}\left(\int_{0}^{\eta} \frac{(\eta-s)^{\alpha-p-1}}{\Gamma(\alpha-p)} y(s) d s-\gamma \int_{0}^{1} \frac{(1-s)^{\alpha-p-1}}{\Gamma(\alpha-p)} y(s) d s\right) \\
\left.c_{0}=-\frac{2 \eta^{2}(\beta+\gamma-1)}{2(1-\eta) Q_{1}} \int_{0}^{\eta}\left(\int_{0}^{s} \frac{(s-\tau)^{\alpha-1}}{\Gamma(\alpha)} y(\tau) d \tau\right) d s+\frac{1}{1-\eta} \int_{0}^{\eta} \int_{0}^{s} \frac{(s-\tau)^{\alpha-1}}{\Gamma(\alpha)} y(\tau) d \tau\right) d s \\
\quad-\frac{\left(\eta^{2}\left[\eta^{3}(\beta+\gamma-1)+3\left(\gamma-\eta^{2}\right)(1-\eta)\right]-\eta^{3} Q_{1}\right) M_{1}}{2(1-\eta) Q_{1}}\left[I^{\alpha-p} y(\eta)-\gamma I^{\alpha-p} y(1)\right] \\
+\frac{\eta^{2}}{Q_{1}}\left[\int_{0}^{\eta} \frac{(\eta-s)^{\alpha-1}}{\Gamma(\alpha)} y(s) d s-\gamma \int_{0}^{1} \frac{(1-s)^{\alpha-1}}{\Gamma(\alpha)} y(s) d s\right]
\end{aligned}
$$

and

$$
\begin{aligned}
c_{1}= & \frac{-2(\beta+\gamma-1)}{Q_{1}} \int_{0}^{\eta}\left(\int_{0}^{s} \frac{(s-\tau)^{\alpha-1}}{\Gamma(\alpha)} y(\tau) d \tau\right) d s-\frac{\left(\eta^{3}(\beta+\gamma-1)+3\left(\gamma-\eta^{2}\right)(1-\eta)\right) M_{1}}{3 Q_{1}}\left[I^{\alpha-p} y(\eta)-\gamma I^{\alpha-p} y(1)\right] \\
& +\frac{2(1-\eta)}{Q_{1}}\left[\int_{0}^{\eta} \frac{(\eta-s)^{\alpha-1}}{\Gamma(\alpha)} y(s) d s-\gamma \int_{0}^{1} \frac{(1-s)^{\alpha-1}}{\Gamma(\alpha)} y(s) d s\right]
\end{aligned}
$$


where

$$
I^{\alpha-p} y(\eta)-\gamma I^{\alpha-p} y(1)=\int_{0}^{\eta} \frac{(\eta-s)^{\alpha-p-1}}{\Gamma(\alpha-p)} y(s) d s-\gamma \int_{0}^{1} \frac{(1-s)^{\alpha-p-1}}{\Gamma(\alpha-p)} y(s) d s .
$$

Substituting the values of constants $c_{0}, c_{1}$ and $c_{2}$ in (3.6), we get (3.5). The proof is complete.

Throughout the paper, we let

$$
\begin{aligned}
& M=\frac{\Gamma(3-p)}{\left|\gamma-\eta^{2}-p\right|} \neq 0,|\beta+\gamma-1| \neq 0,\left|\gamma-\eta^{2}\right| \neq 0, Q=\left|2(1-\eta)(\gamma-\eta)+\eta^{2}\right| \beta+\gamma-1|| \neq 0 \\
& A(t)=|\beta+\gamma-1|\left(\eta^{2}+2(1-\eta) t\right)
\end{aligned}
$$

and

$$
B(t)=\left(\eta^{3}|\beta+\gamma-1|+3\left|\gamma-\eta^{2}\right|(1-\eta)\right)\left(\eta^{2}+2(1-\eta) t\right)-Q\left(\eta^{3}+3(1-\eta) t^{2}\right)
$$

The following inequalities hold:

$$
\begin{aligned}
& |A(t)| \leq|\beta+\gamma-1|\left(\eta^{2}+2(1-\eta)\right)=A_{1}, \\
& |B(t)| \leq\left|\left(\eta^{3}|\beta+\gamma-1|+3\left|\gamma-\eta^{2}\right|(1-\eta)\right)\left(\eta^{2}+2(1-\eta)\right)-Q\left(\eta^{3}+3(1-\eta)\right)\right|=B_{1}, \\
& \left|A^{\prime}(t)\right| \leq 2|\beta+\gamma-1|(1-\eta)=A_{1}^{\prime},
\end{aligned}
$$

and

$$
\left|B^{\prime}(t)\right| \leq 2(1-\eta)\left|\left(\eta^{3}|\beta+\gamma-1|+3\left|\gamma-\eta^{2}\right|(1-\eta)\right)-3 Q\right|=B_{1}^{\prime} .
$$

To simplify the proofs in the forthcoming theorems, we establish the bounds for the integrals arising in the sequel.

Lemma 3.2. For $y \in C([0,1], \mathbb{R})$, we have

$$
\left|\int_{0}^{\eta}\left(\int_{0}^{s} \frac{(s-\tau)^{\alpha-1}}{\Gamma(\alpha)} y(\tau) d \tau\right) d s\right| \leq \frac{\eta^{\alpha+1}}{\Gamma(\alpha+2)}\|y\|
$$

Proof. Obviously,

$$
\int_{0}^{s} \frac{(s-\tau)^{\alpha-1}}{\Gamma(\alpha)} d \tau=\left[-\frac{(s-\tau)^{\alpha}}{\Gamma(\alpha)}\right]_{0}^{s}=\frac{s^{\alpha}}{\alpha \Gamma(\alpha)}=\frac{s^{\alpha}}{\Gamma(\alpha+1)}
$$

Hence

$$
\left|\int_{0}^{\eta}\left(\int_{0}^{s} \frac{(s-\tau)^{\alpha-1}}{\Gamma(\alpha)} y(\tau) d \tau\right) d s\right| \leq\|y\| \int_{0}^{\eta} \frac{s^{\alpha}}{\Gamma(\alpha+1)} d s=\frac{\eta^{\alpha+1}}{\Gamma(\alpha+2)}\|y\| .
$$

For the sake of brevity, we set

$$
\triangle_{1}=\frac{\eta^{\alpha+1}}{(1-\eta) \Gamma(\alpha+2)}+\frac{A_{1} \eta^{\alpha+1}}{Q(1-\eta) \Gamma(\alpha+2)}+\frac{M B_{1}\left(\eta^{\alpha-p}+\gamma\right)}{(1-\eta) Q \Gamma(\alpha-p+1)}+\frac{A_{1}\left(\eta^{\alpha}+\gamma\right)}{Q|\beta+\gamma-1| \Gamma(\alpha+1)}+\frac{1}{\Gamma(\alpha+1)}
$$

and

$$
\triangle_{2}=\frac{A_{1}^{\prime} \eta^{\alpha+1}}{Q(1-\eta) \Gamma(\alpha+2)}+\frac{M B_{1}^{\prime}\left(\eta^{\alpha-p}+\gamma\right)}{(1-\eta) Q \Gamma(\alpha-p+1)}+\frac{A_{1}^{\prime}\left(\eta^{\alpha}+\gamma\right)}{Q|\beta+\gamma-1| \Gamma(\alpha+1)}+\frac{1}{\Gamma(\alpha)}
$$

An element $u \in A C^{2}([0,1, \mathbb{R}])$ is called a solution of the problem (1.1) whenever it satisfies the integral boundary conditions and there exists a function $y \in S_{F, u}$ such that

$$
\begin{aligned}
u(t)= & \int_{0}^{t} \frac{(t-s)^{\alpha-1}}{\Gamma(\alpha)} y(s) d s+\frac{1}{1-\eta} \int_{0}^{\eta}\left(\int_{0}^{s} \frac{(s-\tau)^{\alpha-1}}{\Gamma(\alpha)} y(\tau) d \tau\right) d s-\frac{B(t) M}{6(1-\eta) Q}\left[\int_{0}^{\eta} \frac{(\eta-s)^{\alpha-p-1}}{\Gamma(\alpha-p)} y(s) d s-\gamma \int_{0}^{1} \frac{(1-s)^{\alpha-p-1}}{\Gamma(\alpha-p)} y(s) d s\right] \\
& +\frac{A(t)}{Q|\beta+\gamma-1|}\left[\int_{0}^{\eta} \frac{(\eta-s)^{\alpha-1}}{\Gamma(\alpha)} y(s) d s-\gamma \int_{0}^{1} \frac{(1-s)^{\alpha-1}}{\Gamma(\alpha)} y(s) d s .\right]-\frac{A(t)}{Q(1-\eta)} \int_{0}^{\eta}\left(\int_{0}^{s} \frac{(s-\tau)^{\alpha-1}}{\Gamma(\alpha)} y(\tau) d \tau\right) d s .
\end{aligned}
$$

for all $t \in J$.

For investigation of the problem (1.1) - (1.2) we provide two different methods. 
Theorem 3.3. . Suppose that $F: J \times \mathbb{R} \times \mathbb{R} \rightarrow P_{c p, c v}(\mathbb{R})$ is $L^{1}$-Caratheodory multifunction and there exist a bounded continuous nondecreasing map $\psi:[0, \infty) \rightarrow(0, \infty)$ and a continuous function $p: J \rightarrow(0, \infty)$ such that $\left\|F(t, u(t)), u^{\prime}(t)\right\|=\sup \left\{|v|: v \in F\left(t, u(t), u^{\prime}(t)\right) \leq p(t) \psi(\|u\|)\right\}$, for all $t \in J$ and $u \in X$. Then the inclusion problem (1.1) - (1.2) has at least one solution.

Proof. Define the operator

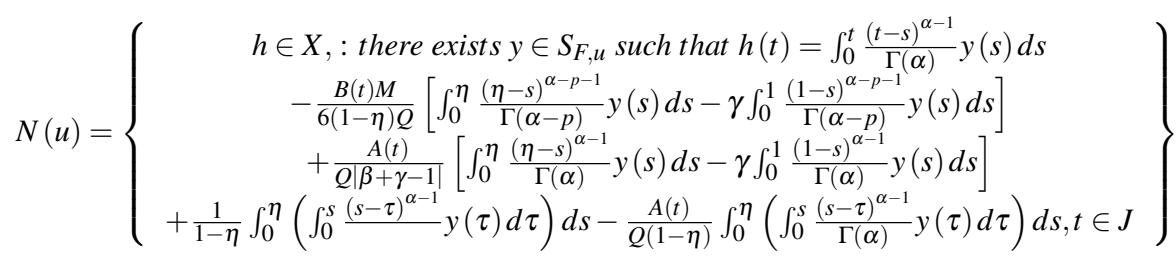

We show that the operator $N$ has a fixed point. First, we show that $N$ maps bounded sets of $X$ into bounded sets. Suppose that $r>0$ and $B_{r}=\{u \in X:\|u\| \leq r\}$. Let $u \in B_{r}$ and $h \in N(u)$. Choose $v \in S_{F, u}$ such that $h(t)$ defined above for almost all $t \in J$. Thus

$$
\begin{aligned}
|h(t)| \leq & \int_{0}^{t} \frac{(t-s)^{\alpha-1}}{\Gamma(\alpha)}|y(s)| d s+\frac{1}{1-\eta} \int_{0}^{\eta}\left(\int_{0}^{s} \frac{(s-\tau)^{\alpha-1}}{\Gamma(\alpha)}|y(\tau)| d \tau\right) d s+\frac{|A(t)|}{Q(1-\eta)} \int_{0}^{\eta}\left(\int_{0}^{s} \frac{(s-\tau)^{\alpha-1}}{\Gamma(\alpha)}|y(\tau)| d \tau\right) d s \\
& +\frac{|B(t)| M}{12(1-\eta) Q}\left[\int_{0}^{\eta} \frac{(\eta-s)^{\alpha-p-1}}{\Gamma(\alpha-p)}|y(s)| d s+\gamma \int_{0}^{1} \frac{(1-s)^{\alpha-p-1}}{\Gamma(\alpha-p)}|y(s)| d s\right] \\
& +\frac{(\gamma-\eta)|A(t)|}{2 Q(\beta+\gamma-1)}\left[\int_{0}^{\eta} \frac{(\eta-s)^{\alpha-1}}{\Gamma(\alpha)}|y(s)| d s+\gamma \int_{0}^{1} \frac{(1-s)^{\alpha-1}}{\Gamma(\alpha)}|y(s)| d s\right] \\
\leq & \Delta_{1}\|p\|_{\infty} \psi(\|u\|),
\end{aligned}
$$

and

$$
\begin{aligned}
\left|h^{\prime}(t)\right| \leq & \int_{0}^{t} \frac{(t-s)^{\alpha-2}}{\Gamma(\alpha-1)}|y(s)| d s+\frac{\left|A^{\prime}(t)\right|}{Q(1-\eta)} \int_{0}^{\eta}\left(\int_{0}^{s} \frac{(s-\tau)^{\alpha-1}}{\Gamma(\alpha)}|y(\tau)| d \tau\right) d s \\
& +\frac{\left|B^{\prime}(t)\right| M}{12(1-\eta) Q}\left[\int_{0}^{\eta} \frac{(\eta-s)^{\alpha-p-1}}{\Gamma(\alpha-p)}|y(s)| d s+\gamma \int_{0}^{1} \frac{(1-s)^{\alpha-p-1}}{\Gamma(\alpha-p)}|y(s)| d s\right] \\
& +\frac{(\gamma-\eta)\left|A^{\prime}(t)\right|}{2 Q(\beta+\gamma-1)}\left[\int_{0}^{\eta} \frac{(\eta-s)^{\alpha-1}}{\Gamma(\alpha)}|y(s)| d s+\gamma \int_{0}^{1} \frac{(1-s)^{\alpha-1}}{\Gamma(\alpha)}|y(s)| d s\right] \\
\leq & \Delta_{2}\|p\|_{\infty} \psi(\|u\|),
\end{aligned}
$$

for all $t \in J$, where $\|p\|_{\infty}=\sup _{t \in J}|p(t)|$.

Hence,

$$
\|h\|=\max _{t \in J}|h(t)|+\max _{t \in J}\left|h^{\prime}(t)\right| \leq\left(\Delta_{1}+\Delta_{2}\right)\|p\|_{\infty} \psi(\|u\|)
$$

Now, we show that $N$ maps bounded sets into equicontinuous subsets of $X$. Let $u \in B_{r}$ and $t_{1}, t_{2} \in J$ with $t_{1}<t_{2}$. Then we have

$$
\begin{aligned}
\left|h\left(t_{2}\right)-h\left(t_{1}\right)\right| \leq & \int_{0}^{t_{2}} \frac{\left(t_{2}-s\right)^{\alpha-1}}{\Gamma(\alpha)}|y(s)| d s+\int_{0}^{t_{1}} \frac{(t-s)^{\alpha-1}}{\Gamma(\alpha)}|y(s)| d s \\
& +\frac{\left|B\left(t_{2}\right)-B\left(t_{1}\right)\right| M}{12(1-\eta) Q}\left[\int_{0}^{\eta} \frac{(\eta-s)^{\alpha-p-1}}{\Gamma(\alpha-p)}|y(s)| d s+\gamma \int_{0}^{1} \frac{(1-s)^{\alpha-p-1}}{\Gamma(\alpha-p)}|y(s)| d s\right] \\
& +\frac{(\gamma-\eta)\left|A\left(t_{2}\right)-A\left(t_{1}\right)\right|}{2 Q(\beta+\gamma-1)}\left[\int_{0}^{\eta} \frac{(\eta-s)^{\alpha-1}}{\Gamma(\alpha)}|y(s)| d s+\gamma \int_{0}^{1} \frac{(1-s)^{\alpha-1}}{\Gamma(\alpha)}|y(s)| d s\right] \\
& +\frac{1}{1-\eta} \int_{0}^{\eta}\left(\int_{0}^{s} \frac{(s-\tau)^{\alpha-1}}{\Gamma(\alpha)}|y(\tau)| d \tau\right) d s+\frac{\left|A\left(t_{2}\right)-A\left(t_{1}\right)\right|}{Q(1-\eta)} \int_{0}^{\eta}\left(\int_{0}^{s} \frac{(s-\tau)^{\alpha-1}}{\Gamma(\alpha)}|y(\tau)| d \tau\right) d s
\end{aligned}
$$




$$
\begin{aligned}
\leq & \int_{0}^{t_{1}} \frac{\left(t_{2}-s\right)^{\alpha-1}-\left(t_{1}-s\right)^{\alpha-1}}{\Gamma(\alpha)}\left[\|p\|_{\infty} \psi(\|u\|)\right] d s+\int_{t_{1}}^{t_{2}} \frac{(t-s)^{\alpha-1}}{\Gamma(\alpha)}\left[\|p\|_{\infty} \psi(\|u\|)\right] d s \\
& +\frac{\left|B\left(t_{2}\right)-B\left(t_{1}\right)\right| M}{12(1-\eta) Q}\left[\int_{0}^{\eta} \frac{(\eta-s)^{\alpha-p-1}}{\Gamma(\alpha-p)}\left[\|p\|_{\infty} \psi(\|u\|)\right] d s+\gamma \int_{0}^{1} \frac{(1-s)^{\alpha-p-1}}{\Gamma(\alpha-p)}\left[\|p\|_{\infty} \psi(\|u\|)\right] d s\right] \\
& +\frac{(\gamma-\eta)\left|A\left(t_{2}\right)-A\left(t_{1}\right)\right|}{2 Q(\beta+\gamma-1)}\left[\int_{0}^{\eta} \frac{(\eta-s)^{\alpha-1}}{\Gamma(\alpha)}\left[\|p\|_{\infty} \psi(\|u\|)\right] d s+\gamma \int_{0}^{1} \frac{(1-s)^{\alpha-1}}{\Gamma(\alpha)}\left[\|p\|_{\infty} \psi(\|u\|)\right] d s\right] \\
& +\frac{1}{1-\eta} \int_{0}^{\eta}\left(\int_{0}^{s} \frac{(s-\tau)^{\alpha-1}}{\Gamma(\alpha)}\left[\|p\|_{\infty} \psi(\|u\|)\right] d \tau\right) d s+\frac{\left|A\left(t_{2}\right)-A\left(t_{1}\right)\right|}{Q(1-\eta)} \int_{0}^{\eta}\left(\int_{0}^{s} \frac{(s-\tau)^{\alpha-1}}{\Gamma(\alpha)}\|p\|_{\infty} \psi(\|u\|) d \tau\right) d s
\end{aligned}
$$

It is seen that $\left|(h)\left(t_{2}\right)-(h)\left(t_{1}\right)\right| \rightarrow 0$, as $t_{2} \rightarrow t_{1}$. Also, we have

$$
\begin{aligned}
& \left|h^{\prime}\left(t_{2}\right)-h^{\prime}\left(t_{1}\right)\right| \leq \int_{0}^{t_{2}} \frac{\left(t_{2}-s\right)^{\alpha-2}}{\Gamma(\alpha-1)}|y(s)| d s+\int_{0}^{t_{1}} \frac{(t-s)^{\alpha-2}}{\Gamma(\alpha-1)}|y(s)| d s \\
& +\frac{\left|B^{\prime}\left(t_{2}\right)-B^{\prime}\left(t_{1}\right)\right| M}{12(1-\eta) Q}\left[\int_{0}^{\eta} \frac{(\eta-s)^{\alpha-p-1}}{\Gamma(\alpha-p)}|y(s)| d s+\gamma \int_{0}^{1} \frac{(1-s)^{\alpha-p-1}}{\Gamma(\alpha-p)}|y(s)| d s\right] \\
& +\frac{(\gamma-\eta)\left|A^{\prime}\left(t_{2}\right)-A^{\prime}\left(t_{1}\right)\right|}{2 Q(\beta+\gamma-1)}\left[\int_{0}^{\eta} \frac{(\eta-s)^{\alpha-1}}{\Gamma(\alpha)}|y(s)| d s+\gamma \int_{0}^{1} \frac{(1-s)^{\alpha-1}}{\Gamma(\alpha)}|y(s)| d s\right] \\
& +\frac{\left|A^{\prime}\left(t_{2}\right)-A^{\prime}\left(t_{1}\right)\right|}{Q(1-\eta)} \int_{0}^{\eta}\left(\int_{0}^{s} \frac{(s-\tau)^{\alpha-1}}{\Gamma(\alpha)}|y(\tau)| d \tau\right) d s \\
& \leq \int_{0}^{t_{1}} \frac{\left(t_{2}-s\right)^{\alpha-2}-\left(t_{1}-s\right)^{\alpha-1}}{\Gamma(\alpha-1)}\left[\|p\|_{\infty} \psi(\|u\|)\right] d s+\int_{t_{1}}^{t_{2}} \frac{(t-s)^{\alpha-2}}{\Gamma(\alpha-1)}\left[\|p\|_{\infty} \psi(\|u\|)\right] d s \\
& +\frac{\left|B^{\prime}\left(t_{2}\right)-B^{\prime}\left(t_{1}\right)\right| M}{12(1-\eta) Q}\left[\int_{0}^{\eta} \frac{(\eta-s)^{\alpha-p-1}}{\Gamma(\alpha-p)}\left[\|p\|_{\infty} \psi(\|u\|)\right] d s+\gamma \int_{0}^{1} \frac{(1-s)^{\alpha-p-1}}{\Gamma(\alpha-p)}\left[\|p\|_{\infty} \psi(\|u\|)\right] d s\right] \\
& +\frac{(\gamma-\eta)\left|A^{\prime}\left(t_{2}\right)-A^{\prime}\left(t_{1}\right)\right|}{2 Q(\beta+\gamma-1)}\left[\int_{0}^{\eta} \frac{(\eta-s)^{\alpha-1}}{\Gamma(\alpha)}\left[\|p\|_{\infty} \psi(\|u\|)\right] d s+\gamma \int_{0}^{1} \frac{(1-s)^{\alpha-1}}{\Gamma(\alpha)}\left[\|p\|_{\infty} \psi(\|u\|)\right] d s\right] \\
& +\frac{\left|A^{\prime}\left(t_{2}\right)-A^{\prime}\left(t_{1}\right)\right|}{Q(1-\eta)} \int_{0}^{\eta}\left(\int_{0}^{s} \frac{(s-\tau)^{\alpha-1}}{\Gamma(\alpha)}\|p\|_{\infty} \psi(\|u\|) d \tau\right) d s
\end{aligned}
$$

Again, we see that $\left|(h)\left(t_{2}\right)-(h)\left(t_{1}\right)\right| \rightarrow 0$, as $t_{2} \rightarrow t_{1}$. Also, we have $\left\|(h)\left(t_{2}\right)-(h)\left(t_{1}\right)\right\| \rightarrow 0$, as $t_{2} \rightarrow t_{1}$. Thus $N$ is equicontinuous and so $N$ is relatively compact on $B_{r}$. Consequently the Ascoli-Arzela theorem implies that $N$ is compact on $B_{r}$.

Now, we show that $N$ has a closed graph. Let $u_{n} \rightarrow u_{0}, h_{n} \in N\left(u_{n}\right)$ for all $n$ and $h \rightarrow h_{0}$. We prove that $h_{0} \in N\left(u_{0}\right)$. For each $n$ choose $y_{n} \in S_{F, u_{n}}$ such that, for all $t \in J$,

$$
\begin{aligned}
h_{n}(t)= & \int_{0}^{t} \frac{(t-s)^{\alpha-1}}{\Gamma(\alpha)} y_{n}(s) d s+\frac{1}{1-\eta} \int_{0}^{\eta}\left(\int_{0}^{s} \frac{(s-\tau)^{\alpha-1}}{\Gamma(\alpha)} y_{n}(\tau) d \tau\right) d s-\frac{A(t)}{Q(1-\eta)} \int_{0}^{\eta}\left(\int_{0}^{s} \frac{(s-\tau)^{\alpha-1}}{\Gamma(\alpha)} y_{n}(\tau) d \tau\right) d s \\
& -\frac{B(t) M}{12(1-\eta) Q}\left[\int_{0}^{\eta} \frac{(\eta-s)^{\alpha-p-1}}{\Gamma(\alpha-p)} y_{n}(s) d s-\gamma \int_{0}^{1} \frac{(1-s)^{\alpha-p-1}}{\Gamma(\alpha-p)} y_{n}(s) d s\right] \\
& +\frac{(\gamma-\eta) A(t)}{2 Q(\beta+\gamma-1)}\left[\int_{0}^{\eta} \frac{(\eta-s)^{\alpha-1}}{\Gamma(\alpha)} y_{n}(s) d s-\gamma \int_{0}^{1} \frac{(1-s)^{\alpha-1}}{\Gamma(\alpha)} y_{n}(s) d s\right]
\end{aligned}
$$


Consider the continuous linear operator $\theta: L^{1}(J, \mathbb{R}) \rightarrow X$ defined by

$$
\begin{aligned}
\theta(y)(t)= & \int_{0}^{t} \frac{(t-s)^{\alpha-1}}{\Gamma(\alpha)} y(s) d s+\frac{1}{1-\eta} \int_{0}^{\eta}\left(\int_{0}^{s} \frac{(s-\tau)^{\alpha-1}}{\Gamma(\alpha)} y(\tau) d \tau\right) d s-\frac{A(t)}{Q(1-\eta)} \int_{0}^{\eta}\left(\int_{0}^{s} \frac{(s-\tau)^{\alpha-1}}{\Gamma(\alpha)} y(\tau) d \tau\right) d s \\
& -\frac{B(t) M}{12(1-\eta) Q}\left[\int_{0}^{\eta} \frac{(\eta-s)^{\alpha-p-1}}{\Gamma(\alpha-p)} y(s) d s-\gamma \int_{0}^{1} \frac{(1-s)^{\alpha-p-1}}{\Gamma(\alpha-p)} y(s) d s\right] \\
& +\frac{(\gamma-\eta) A(t)}{2 Q(\beta+\gamma-1)}\left[\int_{0}^{\eta} \frac{(\eta-s)^{\alpha-1}}{\Gamma(\alpha)} y(s) d s-\gamma \int_{0}^{1} \frac{(1-s)^{\alpha-1}}{\Gamma(\alpha)} y(s) d s\right]
\end{aligned}
$$

By using Lemma 2.13, $\theta \circ S_{F}$ is closed graph operator. Since $u_{n} \rightarrow u$ and $h_{n} \in \theta\left(S_{F, u_{n}}\right)$ for all $n \in \mathbb{N}$, there exist $y_{0} \in S_{F, u_{0}}$ such that

$$
\begin{aligned}
h_{0}(t)= & \int_{0}^{t} \frac{(t-s)^{\alpha-1}}{\Gamma(\alpha)} y_{0}(s) d s+\frac{1}{1-\eta} \int_{0}^{\eta}\left(\int_{0}^{s} \frac{(s-\tau)^{\alpha-1}}{\Gamma(\alpha)} y_{0}(\tau) d \tau\right) d s-\frac{A(t)}{Q(1-\eta)} \int_{0}^{\eta}\left(\int_{0}^{s} \frac{(s-\tau)^{\alpha-1}}{\Gamma(\alpha)} y_{0}(\tau) d \tau\right) d s \\
& -\frac{B(t) M}{12(1-\eta) Q}\left[\int_{0}^{\eta} \frac{(\eta-s)^{\alpha-p-1}}{\Gamma(\alpha-p)} y_{0}(s) d s-\gamma \int_{0}^{1} \frac{(1-s)^{\alpha-p-1}}{\Gamma(\alpha-p)} y_{0}(s) d s\right] \\
& +\frac{(\gamma-\eta) A(t)}{2 Q(\beta+\gamma-1)}\left[\int_{0}^{\eta} \frac{(\eta-s)^{\alpha-1}}{\Gamma(\alpha)} y_{0}(s) d s-\gamma \int_{0}^{1} \frac{(1-s)^{\alpha-1}}{\Gamma(\alpha)} y_{0}(s) d s\right]
\end{aligned}
$$

Thus $N$ has a closed graph.

Now we show that $N(u)$ is convex for all $u \in X$. Let $h_{1}, h_{2} \in N(u)$ and $w \in[0,1]$. Choose $y_{1}, y_{2} \in S_{F, u}$. Then

$$
\begin{aligned}
w h_{1}(t)-(1-w) h_{2}(t)= & \left.\int_{0}^{t} \frac{(t-s)^{\alpha-1}}{\Gamma(\alpha)}\left[w y_{1}(s)-(1-w) y_{2}(s)\right] d s+\frac{1}{1-\eta} \int_{0}^{\eta} \int_{0}^{s} \frac{(s-\tau)^{\alpha-1}}{\Gamma(\alpha)}\left[y_{1}(\tau)-(1-w) y_{2}(\tau)\right] d \tau\right) d s \\
& -\frac{A(t)}{Q(1-\eta)} \int_{0}^{\eta}\left[\int_{0}^{s} \frac{(s-\tau)^{\alpha-1}}{\Gamma(\alpha)}\left[y_{1}(\tau)-(1-w) y_{2}(\tau)\right] d \tau\right) d s \\
& -\frac{B(t) M}{12(1-\eta) Q}\left[\int_{0}^{\eta} \frac{(\eta-s)^{\alpha-p-1}}{\Gamma(\alpha-p)}\left[w y_{1}(s)-(1-w) y_{2}(s)\right] d s-\gamma \int_{0}^{1} \frac{(1-s)^{\alpha-p-1}}{\Gamma(\alpha-p)}\left[w y_{1}(s)-(1-w) y_{2}(s)\right] d s\right] \\
& +\frac{(\gamma-\eta) A(t)}{2 Q(\beta+\gamma-1)}\left[\int_{0}^{\eta} \frac{(\eta-s)^{\alpha-1}}{\Gamma(\alpha)}\left[w y_{1}(s)-(1-w) y_{2}(s)\right] d s-\gamma \int_{0}^{\left.\frac{(1-s)^{\alpha-1}}{\Gamma(\alpha)}\left[w y_{1}(s)-(1-w) y_{2}(s)\right] d s\right]}\right.
\end{aligned}
$$

for all $t \in J$. Since $F$ has convex values, $S_{F, u}$ is convex and so $w h_{1}(t)-(1-w) h_{2}(t) \in N(u)$.

If there exists $\lambda \in(0,1)$ such that $u \in \lambda N(u)$ then there exists $y \in S_{F, u}$ such that

$$
\begin{aligned}
u(t)= & \int_{0}^{t} \frac{(t-s)^{\alpha-1}}{\Gamma(\alpha)} y(s) d s+\frac{1}{1-\eta} \int_{0}^{\eta}\left(\int_{0}^{s} \frac{(s-\tau)^{\alpha-1}}{\Gamma(\alpha)} y(\tau) d \tau\right) d s-\frac{A(t)}{Q(1-\eta)} \int_{0}^{\eta}\left(\int_{0}^{s} \frac{(s-\tau)^{\alpha-1}}{\Gamma(\alpha)} y(\tau) d \tau\right) d s \\
& -\frac{B(t) M}{12(1-\eta) Q}\left[\int_{0}^{\eta} \frac{(\eta-s)^{\alpha-p-1}}{\Gamma(\alpha-p)} y(s) d s-\gamma \int_{0}^{1} \frac{(1-s)^{\alpha-p-1}}{\Gamma(\alpha-p)} y(s) d s\right] \\
& +\frac{(\gamma-\eta) A(t)}{2 Q(\beta+\gamma-1)}\left[\int_{0}^{\eta} \frac{(\eta-s)^{\alpha-1}}{\Gamma(\alpha)} y(s) d s-\gamma \int_{0}^{1} \frac{(1-s)^{\alpha-1}}{\Gamma(\alpha)} y(s) d s\right]
\end{aligned}
$$

for almost all $t \in J$. Choose $L>0$ such that $\frac{L}{\left(\Delta_{1}+\Delta_{2}\right)\|p\|_{\infty} \psi(\|u\|)}>1$ for all $u \in X$. Thus $\|u\|<L$. Now, put $U=\{u \in X:\|u\|<L+1\}$. Note there are no $u \in \partial U$ and $\lambda \in(0,1)$ such that $u \in \lambda N(u)$ and the operator $N: \bar{U} \rightarrow P_{c p, c v}(\bar{U})$ is upper semi-continuous because it is completely continuous. Now, by using Lemma $2.12, N$ has fixed point in $\bar{U}$ which is solution of the inclusion problem (1.1). This complete the proof.

We provide another result about the existence of solutions for the problem $(1.1)-(1.2)$ by changing the assumptions of convex values for multifunction.

Theorem 3.4. Let $m \in C\left(J, \mathbb{R}^{+}\right)$be such that $\|m\|_{\infty}\left(\Delta_{1}+\Delta_{2}\right)<1$.

Suppose that $F: J \times \mathbb{R} \times \mathbb{R} \times \mathbb{R} \rightarrow P_{c p}(\mathbb{R})$ is an integrable bounded multifunction such that the map $t \longmapsto F(t, u, v, w)$ is measurable and $H_{d}\left(F\left(t, u_{1}, u_{2}, u_{3}\right), F\left(t, v_{1}, v_{2}, v_{3}\right)\right) \leq m(t)\left(\left|u_{1}-v_{1}\right|+\left|u_{2}-v_{2}\right|+\left|u_{3}-v_{3}\right|\right)$ for almost all $t \in J$ and $u, v, w, u_{1}, u_{2}, u_{3}, v_{1}, v_{2}, v_{3} \in \mathbb{R}$. Then the problem (1.1) - (1.2) has a solution. 
Proof. Note that, the multivalued map $t \longmapsto F(t, u(t), v(t), w(t))$ is measurable and closed for all $u \in X$. Hence, it has a measurable selection and so the set $S_{F, u}$ is nonempty. Now, consider the operator $N: X \rightarrow 2^{X}$ defined by

$$
N(u)=\left\{h \in X: \text { there exists } v \in S_{F, u} \text { such that } h(t)=u(t), t \in J\right\}
$$

where $u(t)$ defined by (3.5), for all $t \in J$.

First, we show that $N(u)$ is a closed subset of $X$ for all $u \in X$. Let $u \in X$ and $\left\{u_{n}\right\}_{n>1}$ be a sequence in $N(u)$ with $u_{n} \rightarrow u$ for each $n$, such that

$$
\begin{aligned}
u_{n}(t)= & \int_{0}^{t} \frac{(t-s)^{\alpha-1}}{\Gamma(\alpha)} y_{n}(s) d s+\frac{1}{1-\eta} \int_{0}^{\eta}\left(\int_{0}^{s} \frac{(s-\tau)^{\alpha-1}}{\Gamma(\alpha)} y_{n}(\tau) d \tau\right) d s-\frac{A(t)}{Q(1-\eta)} \int_{0}^{\eta}\left(\int_{0}^{s} \frac{(s-\tau)^{\alpha-1}}{\Gamma(\alpha)} y_{n}(\tau) d \tau\right) d s \\
& -\frac{B(t) M}{12(1-\eta) Q}\left[\int_{0}^{\eta} \frac{(\eta-s)^{\alpha-p-1}}{\Gamma(\alpha-p)} y_{n}(s) d s-\gamma \int_{0}^{1} \frac{(1-s)^{\alpha-p-1}}{\Gamma(\alpha-p)} y_{n}(s) d s\right] \\
& +\frac{(\gamma-\eta) A(t)}{2 Q(\beta+\gamma-1)}\left[\int_{0}^{\eta} \frac{(\eta-s)^{\alpha-1}}{\Gamma(\alpha)} y_{n}(s) d s-\gamma \int_{0}^{1} \frac{(1-s)^{\alpha-1}}{\Gamma(\alpha)} y_{n}(s) d s\right]
\end{aligned}
$$

for almost all $t \in J$. Since $F$ has compact values, $\left\{y_{n}\right\}_{n \geq 1}$ has a subsequence which converges to some $y \in L^{1}(J, \mathbb{R})$. It is easy to check that $y \in S_{F, u}$ and $u_{n}(t) \rightarrow u(t)$ for all $t \in J$. This implies that $u \in N(u)$. Thus the multifunction $N$ has closed values.

Now, we show that $N$ is a contractive multifunction with constant

$l=\|m\|_{\infty}\left(\Delta_{1}+\Delta_{2}\right)<1$.

Let $u, v \in X$ and $h_{1} \in N(v)$. Choose $y_{1} \in S_{F, v}$ such that

$$
\begin{aligned}
h_{1}(t)= & \int_{0}^{t} \frac{(t-s)^{\alpha-1}}{\Gamma(\alpha)} y_{1}(s) d s+\frac{1}{1-\eta} \int_{0}^{\eta}\left(\int_{0}^{s} \frac{(s-\tau)^{\alpha-1}}{\Gamma(\alpha)} y_{1}(\tau) d \tau\right) d s-\frac{A(t)}{Q(1-\eta)} \int_{0}^{\eta}\left(\int_{0}^{s} \frac{(s-\tau)^{\alpha-1}}{\Gamma(\alpha)} y_{1}(\tau) d \tau\right) d s \\
& -\frac{B(t) M}{12(1-\eta) Q}\left[\int_{0}^{\eta} \frac{(\eta-s)^{\alpha-p-1}}{\Gamma(\alpha-p)} y_{1}(s) d s-\gamma \int_{0}^{1} \frac{(1-s)^{\alpha-p-1}}{\Gamma(\alpha-p)} y_{1}(s) d s\right] \\
& +\frac{(\gamma-\eta) A(t)}{2 Q(\beta+\gamma-1)}\left[\int_{0}^{\eta} \frac{(\eta-s)^{\alpha-1}}{\Gamma(\alpha)} y_{1}(s) d s-\gamma \int_{0}^{1} \frac{(1-s)^{\alpha-1}}{\Gamma(\alpha)} y_{1}(s) d s\right]
\end{aligned}
$$

for almost all $t \in J$. Since

$H_{d}\left(F\left(t, u(t), u^{\prime}(t)\right), F\left(t, v(t), v^{\prime}(t)\right)\right) \leq m(t)\left(|u(t)-v(t)|+\left|u^{\prime}(t)-v^{\prime}(t)\right|\right)$ for almost all $t \in J$ there exists $w \in F\left(t, u(t), u^{\prime}(t)\right)$ such that

$$
\left|y_{1}(t)-w\right| \leq m(t)\left(|u(t)-v(t)|+\left|u^{\prime}(t)-v^{\prime}(t)\right|\right)
$$

for almost all $t \in J$.

Define the multifunction $U: J \rightarrow 2^{\mathbb{R}}$ by

$$
U(t)=\left\{w \in \mathbb{R}:\left|y_{1}(t)-w\right| \leq m(t)\left(|u(t)-v(t)|+\left|u^{\prime}(t)-v^{\prime}(t)\right|\right) \text { for almost all } t \in J\right\} .
$$

It is easy to chek that the multifunction $U(\cdot) \cap F(\cdot), u(\cdot), u^{\prime}(\cdot)$ is measurable. Thus, we can choose $y_{2} \in S_{F, u}$ such that

$$
\left|y_{1}(t)-y_{2}(t)\right| \leq m(t)\left(|u(t)-v(t)|+\left|u^{\prime}(t)-v^{\prime}(t)\right|\right),
$$

for almost all $t \in J$. Now, consider $h_{2} \in N(u)$ which is defined by

$$
\begin{aligned}
h_{2}(t)= & \int_{0}^{t} \frac{(t-s)^{\alpha-1}}{\Gamma(\alpha)} y_{2}(s) d s+\frac{1}{1-\eta} \int_{0}^{\eta}\left(\int_{0}^{s} \frac{(s-\tau)^{\alpha-1}}{\Gamma(\alpha)} y_{2}(\tau) d \tau\right) d s-\frac{A(t)}{Q(1-\eta)} \int_{0}^{\eta}\left(\int_{0}^{s} \frac{(s-\tau)^{\alpha-1}}{\Gamma(\alpha)} y_{2}(\tau) d \tau\right) d s \\
& -\frac{B(t) M}{12(1-\eta) Q}\left[\int_{0}^{\eta} \frac{(\eta-s)^{\alpha-p-1}}{\Gamma(\alpha-p)} y_{2}(s) d s-\gamma \int_{0}^{1} \frac{(1-s)^{\alpha-p-1}}{\Gamma(\alpha-p)} y_{2}(s) d s\right] \\
& +\frac{(\gamma-\eta) A(t)}{2 Q(\beta+\gamma-1)}\left[\int_{0}^{\eta} \frac{(\eta-s)^{\alpha-1}}{\Gamma(\alpha)} y_{2}(s) d s-\gamma \int_{0}^{1} \frac{(1-s)^{\alpha-1}}{\Gamma(\alpha)} y_{2}(s) d s\right]
\end{aligned}
$$


Hence, we get

$$
\begin{aligned}
\left|h_{1}(t)-h_{2}(t)\right| \leq & \int_{0}^{t} \frac{(t-s)^{\alpha-1}}{\Gamma(\alpha)}\left|y_{1}(s)-y_{2}(s)\right| d s+\frac{1}{1-\eta} \int_{0}^{\eta}\left(\int_{0}^{s} \frac{(s-\tau)^{\alpha-1}}{\Gamma(\alpha)}\left|y_{1}(\tau)-y_{2}(\tau)\right| d \tau\right) d s \\
& +\frac{|A(t)|}{Q(1-\eta)} \int_{0}^{\eta}\left(\int_{0}^{s} \frac{(s-\tau)^{\alpha-1}}{\Gamma(\alpha)}\left|y_{1}(\tau)-y_{2}(\tau)\right| d \tau\right) d s \\
& +\frac{|B(t)| M}{12(1-\eta) Q}\left[\int_{0}^{\eta} \frac{(\eta-s)^{\alpha-p-1}}{\Gamma(\alpha-p)}\left|y_{1}(s)-y_{2}(s)\right| d s+\gamma \int_{0}^{1} \frac{(1-s)^{\alpha-p-1}}{\Gamma(\alpha-p)}\left|y_{1}(s)-y_{2}(s)\right| d s\right] \\
& +\frac{(\gamma-\eta)|A(t)|}{2 Q(\beta+\gamma-1)}\left[\int_{0}^{\eta} \frac{(\eta-s)^{\alpha-1}}{\Gamma(\alpha)}\left|y_{1}(s)-y_{2}(s)\right| d s+\gamma \int_{0}^{1} \frac{(1-s)^{\alpha-1}}{\Gamma(\alpha)}\left|y_{1}(s)-y_{2}(s)\right| d s\right] \\
\leq & \Delta_{1}\|m\|_{\infty}\|u-v\|,
\end{aligned}
$$

and so $\left\|h_{1}-h_{2}\right\| \leq\left(\triangle_{1}+\triangle_{2}\right)\|m\|_{\infty}\|u-v\|=l\|u-v\|$. This implies that the multifunction $N$ is a contraction which closed values. Thus, by using the result of Covitz and Nadler, $N$ has a fixed point which is solution for the inclusion problem.

We construct two examples to illustrate the applicability of the results presented.

Example 3.5. Consider the problem

$$
{ }^{c} D^{3} u(t) \in F(t, u, v), \quad t \in[0,1],
$$

subject to the three-point boundary conditions

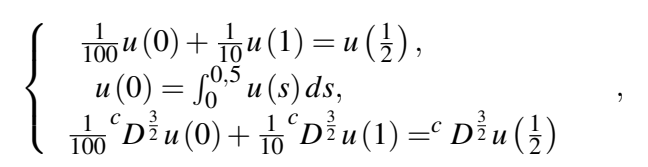

where $\eta=0,5, \beta=0,01, \gamma=0,1, p=1,5$ and $F(t, u, v):[0,1] \times \mathbb{R}^{2} \rightarrow 2^{\mathbb{R}}$ multivalued map given by

$$
u \longmapsto F(t, u, v)=\left(\left(\frac{3+t^{2}}{4}\right)\left(\frac{|u|}{1+u}+\sin (v)\right), \frac{|u|^{3}}{2\left(1+|u|^{3}\right)}+5 t^{3}+4\right), u, v \in \mathbb{R}
$$

verifying $\left(H_{1}\right)$.

Obviously, for $f \in F$, we have

$$
|f|=\max \left(\left(\frac{3+t^{2}}{4}\right)\left(\frac{|u|}{1+|u|}+\sin (v)\right), \frac{|u|^{3}}{2\left(1+|u|^{3}\right)}+5 t^{3}+4\right) \leq \frac{19}{2}, u, v \in \mathbb{R} .
$$

Thus

$$
\|F(t, u)\|=\sup \{|f|: f \in F(t, u, v)\} \leq \frac{19}{2}, u, v \in \mathbb{R}
$$

where $p(t)=1$ and $\psi(t)=\frac{19}{2}$, then one can check that the assumptions of Theorem 3.3 hold. and so the problem (3.15) - (3.16) has at least one solution.

Example 3.6. Consider the problem (3.15) - (3.16), where $\eta=0,5, \beta=0,01, \gamma=0,1, p=1,5$ and $F(t, u, v):[0,1] \times \mathbb{R}^{2} \rightarrow 2^{\mathbb{R}}$ multivalued map given by

$$
u \longmapsto F(t, u, v)=\left(0, \frac{t|u|}{2(1+|u|)}+\frac{|v|^{3}}{2\left(1+|v|^{3}\right)}\right), u, v \in \mathbb{R} .
$$

Obviously,

$$
H_{d}\left(F\left(t, u_{1}, u_{2}\right), F\left(t, v_{1}, v_{2}\right)\right) \leq\left(\frac{t}{2}+\frac{1}{2}\right) \sum_{i=1}^{2}\left|u_{i}-v_{i}\right|, u, v \in \mathbb{R}, t \in[0,1]
$$

If $m(t)=\frac{t}{2}+\frac{1}{2}$ for all $t \in[0,1] H_{d}\left(F\left(t, u_{1}, u_{2}\right), F\left(t, v_{1}, v_{2}\right)\right) \leq m(t) \sum_{i=1}^{2}\left|u_{i}-v_{i}\right|$.

On the other hand, it can be easily found that $M=1,4597546147, Q=\frac{9}{400}, \Delta_{1} \cong 0,4141664514$ and $\Delta_{2} \cong 0$ ?9758011659.

Finally, since $\|m\|_{\infty}\left(\triangle_{1}+\triangle_{2}\right) \cong 0,143492<1$, thus all assumptions of Theorem 3.4 are satisfied. Hence, The inclusion problem has at least one solution. 


\section{References}

[1] A. Lasota and Z. Opial, An application of the Kakutani-Ky Fan theorem in the theory of ordinary differential equations, Bull. Acad. Pol. Sci. Set. Sci. Math. Astronom. Phy. 13 (1965), 781-786.

[2] A. Ral, V. Gupta and R. P. Agarwal, Applications of q-calculus in operator theory., Springer, New York, (2013).

[3] A. Granas and J. Dugundji, Fixed Point Theory., Springer-Verlag, New York, 2005.

[4] A. A. Kilbas, H. M. Srivastava and J. J. Trijullo, Theory and applications of fractional differential equations, Elsevier Science b. V., Amsterdam, (2006).

[5] A. Ral, V. Gupta and R. P. Agarwal, Applications of q-calculus in operator theory., Springer, New York, (2013).

[6] A. Rezaigia and S. Kelaiaia, Existence results for third-order differential inclusion with three-point boundary value problems, Acta. Math. Univ. Comenianae, 2 (2016), 311-318.

[7] A. M. A. EL-Sayed, F. M. Nesreen and EL-Haddade, Existence of integrable solutions for a functional integral inclusion, Diff. Equa. Cont. Proce, 3 (2017).

[8] A. Cernia, Existence of solutions for a certain boundary value problem associated to a fourth-order differential inclusion, Inter. Jour. Anal. Appl. Vol 14, 1 (2017), 27-33.

[9] B. Ahmad, J. S. Ntouyas and I. Purnaras, Existence results for nonlocal boundary value problems of nonlinear fractional q-difference equations, Adv. Differ. Equ-NY, 2012 (2012), 140, 15 pages.

[10] C. R. Adamas, On the linear ordinary q-difference equation, Ann. Math, 30 (1928), 195-205.

[11] D. Baleanu, H. Mohammadi and S. H. Rezapour, The existence of solutions for a nonlinear mixed problem of singular fractional equations, Adv. Difference Equa. 2013 (2013), 12 pages.

[12] H. Covitz and S. B. Jr. Nadler, Multivalued contraction mappings in generalized metric spaces, Israel J. Math. 8 (1970), 5-11.

[13] H. H. Alsulami, S. K. Ntouyas, S. A. Al-Mezel, B. Ahmad and A. Alsaedi, A study of third-order single-valued and multi-valued problems with integral boundary conditions, Bound. Value. Prob. (2015), 20 pages.

[14] J. P. Aubin and A. Cellina, Differential Inclusions, Springer-Verlag, 2012.

[15] K. Demling, Multivalued Differential equations, Walter De Gryter, Berlin-New-York 1982.

[16] L. Gorniewcz, Topological Fixed Point Theory of Multivalued Map pings, Mathematics and Its Applications vol. 495, Kluwer Academic Publishers, Dordrecht 1999.

[17] M. Kisielewicz, Differential Inclusions and Optimal Control, Mathematics and Its Applications (East European Series), vol. 44, Kluwer Academic Publishers, Dordrecht, The Netherlands, 1991.

[18] N. Bouteraa and S. Benaicha, Existence of solutions for three-point boundary value problem for nonlinear fractional differential equations, Analele Universitatii Oradea Fasc. Matematica, Tom XXIV (2017), Issue No. 2, 109-119.

[19] N. Bouteraa, S. Benaicha and H. Djourdem, Positive solutions for nonlinear fractional differential equation with nonlocal boundary conditions, Universal Journal of Mathematics and Applications. 1 (2018), 39-45.

[20] N. Bouteraa and S. Benaicha, The uniqueness of positive solution for nonlinear fractional differential equation with nonlocal boundary conditions, Analele Universitatii Oradea Fasc. Matematica, Volume 25 (2018), Issue No. 2, To appear.

[21] O. Agrawal, Some generalized fractional calculus operators and their applications in integral equations, Fract. Cal. Appl. Anal. 15 (2012), 700-711.

[22] P. M. Rajkovic, S. D. Marancovic and M. S. Stankovic, On q-analogues of Caputo derivative and Mettag-Leffter function, Frac. Calc. Appl. Anal. 10 (2007), 359-373.

[23] R. P. Agarwal, D. Baleanu, V. Hedayati and S. H. Rezapour, Two fractional derivative inclusion problems via integral boundary condition, Appl. Math. Comput. 257 (2015), 205-212.

[24] R. P. Agarwal, S. K. Ntouyas, B. Ahmad and M. S. Alhouthuali, Existence of solutions for integro-differential equations of fractional order with nonlocal tree-point fractional boundary condition, Adv. Difference Equ. 2013 (2013).

[25] R. Ghorbanian, V. Hedayati, M. Postolache and S. H. Rezapour, On a fractional differential inclusion via a new integral boundary condition, J. Inequal. Appl. 2014 (2014), 20 pages.

[26] S. Miller and B. Ross, An introduction to the fractional calculus and fractional differential equations, John Wiley and Sons, Inc. New York, (1993).

[27] S. K. Ntouyas, Existence of solutions for fractional differencial inclusions with integral boundary conditions, Bound. Value Prob. 2015 (2015), 14 pages

[28] S. K. Ntouyas, S. Etemad and J. Tariboon, Existence results for multi-term fractional diffrential inclusions, Adv. Difference Equ. 2015 (2015), 15 pages.

[29] S. K. Ntouyas, S. Etemad, J. Tariboon and W. Sutsutad, Boundary value problems for Riemann-Liouville nonlinear fractional diffrential inclusions with nonlocal Hadamard fractional integral conditions, Medittter. J. Math. 2015 (2015), 16 pages.

[30] S. Etemad, S. K. Ntouyas and J. Tariboon, Existence results for three-point boundary value problems for nonlinear fractional diffrential equations. J. Nonlinear Sci. Appl. 9 (2016).

[31] P. D. Phung and L. X. Truong, On a fractional differential inclusion with integral boundary conditions in Banach space, Fract. Calc. Appl. Anal. 16(3) (2013), 538-558.

[32] R. P. Agarwal and B. Ahmad, Existence theory for anti-periodic boundary value problems of fractional differential equations and inclusions, J. Appl. Math. Comput. 62 (2011).

[33] R. Ghorbanian, V. Hedayati, M. Postolache and S. H. Rezapour, On a fractional differential inclusion via a new integral boundary condition, J. Inequal. Appl. 2014 (2014), 20 pages.

[34] R. P. Agarwal, D. Baleanu, V. Hedayati and S. H. Rezapour, Two fractional derivative inclusion problems via integral boundary condition, Appl. Math. Comput. 257 (2015), 205-212.

[35] S. Hu and N. Papageorgeou, Handbook of Multivalued Analysis, Volume I

[36] S. Suganya and M. M. Arjunan, Existence of Mild Solutions for Impulsive Fractional Integro-Differential Inclusions with State-Dependent Delay, Mathematics. 9 (2017), 2-16.

[37] V. Kac and P. Cheung, Quantum Calculus, Springer, New York, (2002).

[38] V. Berinde and M. Pacurar, The role of the Pompeiu-Hausdorff metric in fixed point theory, Creat. Math. Inform. 22(2) (2013), 35-42. 